\title{
Efecto de un Recubrimiento Comestible y Diferentes Tipos de Empaque en los Atributos Físico-Químicos y Sensoriales de Piña 'Manzana' Mínimamente Procesada
}

\author{
Saúl Dussán-Sarria*, Pedro M. Reyes-Calvache y José I. Hleap-Zapata \\ Universidad Nacional de Colombia, Facultad de Ingeniería, Depto. Ingeniería y Administración, \\ Sede Palmira. A.A. 237. Palmira, Valle del Cauca-Colombia. (e-mail: sdussan@unal.edu.co; \\ pmreyesc@unal.edu.co; jihleap@unal.edu.co)
}

* Autor a quien debe ser dirigida la correspondencia.

Recibido Nov. 19, 2013; Aceptado Ene. 3, 2014; Versión final recibida May. 9, 2014

\begin{abstract}
Resumen
Se evaluó el efecto de recubrimiento comestible y varios tipos de empaque en Piña 'Manzana' mínimamente procesada bajo refrigeración a $5 \pm 1^{\circ} \mathrm{C}$ y $90 \pm 2 \%$ de humedad relativa. Se efectuó un seguimiento de los atributos de calidad cada cuatro días hasta el día 24. Se utilizó el delineamiento experimental completamente al azar con tres repeticiones totalizando ocho tratamientos derivados de cuatro condiciones de empaques y dos de recubrimiento. Se aplicó estadística descriptiva, ANOVA y comparación de medias según Tukey. Se utilizó el software SAS $9.3(p<0.05)$. El recubrimiento comestible tuvo un efecto significativo en la conservación de acuerdo a los valores de porcentaje de acidez titulable AT y de $\mathrm{pH}$. Sin embargo, la piña mínimamente procesada acondicionada al vacío, tratada con ácido ascórbico (1\% v/v), ácido cítrico $(1 \% \mathrm{v} / \mathrm{v})$ y $\mathrm{CaCl}_{2}(1 \% \mathrm{v} / \mathrm{v})$, sin recubrimiento comestible y almacenada bajo refrigeración permitió conservar los atributos de calidad por un periodo 16 días.
\end{abstract}

\section{Effect of Edible Coating and Different Type of Packaging in Physical-Chemical and Sensory Attributes of Fresh-Cut 'Manzana' Pineapple}

\begin{abstract}
The effects of edible coating and of various types of packaging of fresh-cut 'Manzana' pineapple stored under refrigeration at $5 \pm 1{ }^{\circ} \mathrm{C}$ and $90 \pm 2 \%$ of relative humidity were evaluated. The monitoring quality attributes was done every four days until the day 24 . The experimental design used was completely randomized with three replicates with a total of eight treatments derived from four packaging conditions and two of coating. Descriptive statistics, ANOVA and means comparison using Tukey test were applied. The software SAS $9.3(p<0.05)$ was used. The edible coating had significant effect on the conservation according to the titratable acidity TA and $\mathrm{pH}$ values. However, the fresh-cut pineapple in vacuum packing, treated with ascorbic acid $(1 \% \mathrm{v} / \mathrm{v})$, citric acid $(1 \% \mathrm{v} / \mathrm{v})$ and $\mathrm{CaCl}_{2}(1 \% \mathrm{v} / \mathrm{v})$, without edible coating and stored under refrigeration maintained the quality attributes for 16 days.
\end{abstract}

Keywords: Ananas comosus, carnauba wax, cassava starch, edible coating, food packaging 


\section{INTRODUCCIÓN}

La piña es un fruto que se caracteriza por tener altos porcentajes de vitaminas, carbohidratos, fibra y sales minerales siendo el ácido cítrico es el ácidoorgánico predominante(FAO, 2006) ytiene propiedades antiinflamatorias y analgésicas debidas a la bromelina (Pretelt, 2003).Colombia ocupó el puesto 8 en el ranking mundial de producción de piña en frescoen el año 2012, con 551.133t siendo Tailandia el primer productor mundial con 2'650.000 t (FAO, 2014). La piña es un fruto tropical no climatérico que se deteriora rápidamente a condiciones no refrigeradas tanto en estado fresco como cortado (Kader, 2002).El procesamiento mínimo de vegetales frescos, cuando aplicadas técnicas combinadas sinérgicamente, limitael crecimiento microbiano y las reacciones físicas, químicas y bioquímicas. Algunas de estas técnicas son el pre-enfriamiento, la refrigeración en toda la cadena, la atmosfera modificada (AM) y la atmosfera controlada (AC) (Sanjinez-Argandoña et al., 2010).

Las frutas son organismos vivos, por lo tanto, cuanto mayor sea el nivel de procesamiento o manipulación, mayor será el impacto sobre su vida útil (Saavedra et al., 2008). Es importante combinar métodos para mitigar dichos impactos y poder conservar las frutas por más tiempo. El uso deantioxidantes como el ácido ascórbico y cítrico en relaciones 1,0:0,5 y 1,0:1,0\% respectivamente, combinadoscon hipoclorito de sodio conservan el color y no alteran los sabores de la piña MP (Antoniolli et al., 2012). La piña tratada con ácido isoascórbico $(0,1 \mathrm{~mol} / \mathrm{L})$ y ácido ascórbico $(0,05 \mathrm{~mol} / \mathrm{L})$, presenta una reducción significativa en el pardeamiento (González-Aguilaret al., 2004). Se mejora la textura de la fruta utilizando calcio ysecontrola la tasa respiratoria, los desórdenes fisiológicos, la producción de etileno y la podredumbre microbiana (Rahman, 2003; Eduardoet al., 2008).

La aplicación de recubrimientos comestibles ha demostrado ser eficientes al conservar las propiedades de las frutas. Se encontró que el uso de almidón acuoso al $2 \%$, celulosa al $3 \%$, y quitosano al $2 \%$, permitía alargar la vida útil del mango sin alterar su calidad organoléptica (Zambrano et al., 2011). El uso de recubrimientos comestibles a base de almidón de yuca y proteína de soja, reducen la pérdida de peso y conserva las propiedades fisicoquímicas de la fresa(Saavedra y Algecira, 2010). Los envases rígidos son esenciales para reducir deshidratación y daño mecánico durante distribución de frutas y hortalizas frescas y mínimamente procesadas (Kader, 2002). De acuerdo a Rinaldiet al. (2013), la especificación del empaque las verduras frescas cortadas requiere la optimización de los procesos físicos, químicos, bioquímicos y ambientales. La utilización de envases plásticos como empaque se plantea como mecanismo de conservación que proporciona al producto procesado un mayor tiempo de almacenamiento. El objetivo de este estudio fue encontrar la condición agroindustrial que conserve mejor los atributos físico-químicos y sensoriales de piña 'Manzana' mínimamente procesada durante el almacenamiento refrigerado.

\section{METERIALES Y METODOS}

El análisis experimental se llevó a cabo en Laboratorio de Frutas y Hortalizas de la Universidad Nacional de Colombia (Palmira, Valle del Cauca, Colombia, a 1100msnm). Se utilizó piña (Ananascomosus) variedad Manzana en estado de maduración numero 3según tabla de maduración en norma NTC 4102 (ICONTEC, 1997). El fruto se transportó a las instalaciones del Laboratorio, se seleccionaron y clasificaron de acuerdo a la uniformidad en su tamaño y en su estado de madurez ( $50 \%$ de coloración amarilla en la superficie) y se mantuvo en refrigeración a $10^{\circ} \mathrm{C}$ durante 24 horas antes de los cortes.

Preparación de las muestras de piña: Los frutos refrigerados fueron lavados conagua potable e inmersos en agua con hipoclorito de sodio a 100ppm durante $10 \mathrm{~min}$ (Djioua et al., 2009).Se les retiró la cáscara y se cortaron formando cubos de $1,5 \mathrm{~cm}$ de lado, posteriormente los cubos se sumergieron en agua con hipoclorito de sodio a 10 ppmpor 1 minuto yse retiró el exceso de agua por gravedad durante 2 min. Seguidamente los cubos se sumergieron durante 3 minutos en una solución a base de Cloruro de Calcio al 1\%, Ácido Cítrico al 1\%, Ácido Ascórbico al 1\%(Kader, 2002) y se retiróel exceso de humedad.

Preparación y aplicación del recubrimiento comestible: El procedimiento de preparación y cantidad de los componentes utilizadas se realizó en base a la metodología descrita por Dussán-Sarria, et al. (2014).Las muestras de piña cortada una vez aplicado los antioxidantes y el calcio, se dividieron en dos grupos, uno se dejó sin recubrimiento y el otro se sumergió durante 2 minutos en el recubrimiento comestible formado.

Acondicionamiento de los cubos de Piña: Se acondicionaron $150 \mathrm{~g}$ de piña MP en cuatro (4) envases diferentes con las siguientes dimensiones: bandeja de Icopor de $10 \mathrm{~cm} \times 10 \mathrm{~cm}$ envuelta en PVC, cajas de polietileno tereftalato PET de $12 \mathrm{~cm} \times 8 \mathrm{~cm} \times 4 \mathrm{~cm}$, bolsa de polietileno de baja densidad PEBD calibre $70 \mu \mathrm{mde}$ $19 \mathrm{~cm} \times 14 \mathrm{~cm}$ y bolsa de polietileno de baja densidad PEBD calibre $40 \mu$ mde $15 \mathrm{~cm} \times 15 \mathrm{~cm}$. Las respectivas permeabilidades son:film de PVC calibre $14 \mu \mathrm{mal} \mathrm{CO}_{2}$ de $5,1835 \mathrm{~cm}^{3} / \mathrm{m}^{2}$.día y al $\mathrm{O}_{2}$ de $148,0395 \mathrm{~cm}^{3} / \mathrm{m}^{2}$.día,cajas PET al $\mathrm{CO}_{2}$ de $112,3392 \mathrm{~cm}^{3} / \mathrm{m}^{2}$.día y al $\mathrm{O}_{2}$ de $3945,2929 \mathrm{~cm}^{3} / \mathrm{m}^{2}$.día, bolsas de 
PEBDcalibre $70 \mu \mathrm{m}$ para aplicar vacíoal $\mathrm{CO}_{2}$ de $6264,8897 \mathrm{~cm}^{3} / \mathrm{m}^{2}$.día y al $\mathrm{O}_{2}$ de $1163,4795 \mathrm{~cm}^{3} / \mathrm{m}^{2}$. díay bolsas de PEBD calibre $40 \mu \mathrm{m}$ para el tratamiento $\mathrm{BOLSA}$ al $\mathrm{CO}_{2}$ de $10963,5570 \mathrm{~cm}^{3} / \mathrm{m}^{2}$.día y al $\mathrm{O}_{2}$ de $2036,0892 \mathrm{~cm}^{3} / \mathrm{m}^{2}$.día. Todos los empaques iniciaron con una atmosfera modificada pasiva a excepción de las bolsas de PEBD de $70 \mu \mathrm{m}$ que se les aplicó vacío total, es decir, se realizó una atmosfera modificada activa. La piña MP empacada se almacenó en un refrigerador panorámico a Temperatura de $5 \pm 1^{\circ} \mathrm{C}$ y Humedad Relativa de $85 \%$ a $90 \%$ según recomienda Silva et al. (2005) y Kader (2002).

\section{EVALUACIÓN DE ATRIBUTOS}

Se realizaron análisis Físico-químicos y sensoriales a los 0, 4, 8, 12, 16, 20 y 24 días de almacenamiento refrigerado.

\section{Análisis sensorial}

Con la ayuda de 30 jurados no entrenados se evaluó color, apariencia general y aroma usandola siguiente escala numérica: 5 me gusta mucho, 4.0 me gusta, 3.0 ni me gusta ni me disgusta, 2.0 me gusta poco, 1.0 no me gusta. Para considerar el producto aceptable se tuvo en cuenta una nota mayor o igual a 3.0.

\section{Análisis físico-químico}

Los valores de pHse determinaron según la norma NTC 4592 (ICONTEC, 1999a)con un pH-metro digital marca SCHOTT CG-842 de Alemania. Los valores de sólidos solubles (SS) se determinaron siguiendo la norma NTC 4624 (ICONTEC, 1999c)con ayuda de un refractómetro digital (POCKET ATAGO PAL-1, capacidad: $0-53 \%$, Japón). La medición se expresó en Bix. El porcentaje de acidez titulable(AT) se determinó siguiendo la norma NTC 4623 (ICONTEC, 1999b)con el ácido cítrico como el predominante en piña y el índice de madurez (IM) se calculó con la ecuación (1).

Índice de madurez $\%=\frac{{ }^{\circ} \text { Brix }}{\% \text { Acidez }}$

\section{Análisis experimental y estadístico}

Se evaluaron 8 tratamientos derivados de 4 condiciones de envase, y 2 de recubrimiento (con y $\sin$ recubrimiento comestible), designados de la siguiente forma:

\begin{tabular}{lcccc} 
Con Recubrimiento Comestible & PVC-R & VACIO-R & BOLSA-R & PET-R \\
\hline Sin Recubrimiento Comestible & PVC & VACIO & BOLSA & PET
\end{tabular}

La unidad experimental fue el envase conteniendo el fruto cortado. Se utilizó el delineamiento experimental completamente al azar con tres repeticiones y se aplicó análisis estadístico basado en estadística descriptiva, análisis de varianza y comparación de medias utilizando el ensayo Tukey. Estos valores se analizaron a través del software SAS 9.3 con un nivel de significancia $p<0,05$.

\section{RESULTADOS Y DISCUSIÓN}

\section{Análisis sensorial}

En la Figura 1 y Figura 2 se observa un descenso en la aceptación sensorial de la piña MP en todos los tratamientos. La piña MP con recubrimiento y empacada al vacío tuvoaceptación sensorial hasta el día 12 y la piña MP sin recubrimiento y empacada al vacío hasta el día 16 del almacenamiento. Posterior a este día huboaroma desagradable, líquido exudado y el rechazopor los jurados. Los demás tratamientos presentaron aceptación sensorial inferior a los 16 días.

Marrero y Kader (2006) afirman que después de 14 días de almacenada, en la piña aparecen sabores y aromasdesagradables producto del deterioro microbiano. Kader (2002) afirma que la exudación de piña mínimamente procesada es debida a que en el interior de los recipientes de plástico la saturación de vapor de agua no favorece la formación de un gradiente de presión de vapor entre el producto y el espacio vacío.Según Torri et al. (2010) la piña MP pierde el aroma típico por la disminución de compuestos volátiles y por el incremento de compuestos azufrados, polares, alcoholes y cetonas que producen malos olores. Se ha estimado una vida útil para la piña MP de 7 días tratada con cloruro de calcio, cloruro de sodio, AA, AC, quitosano y envasada en cajas de poliestireno (Ediriweera et al., 2012).Martín y Oms (2005) encontraron que en frutas MP la calidad sensorial tiene un límite de una semana de almacenamiento. 


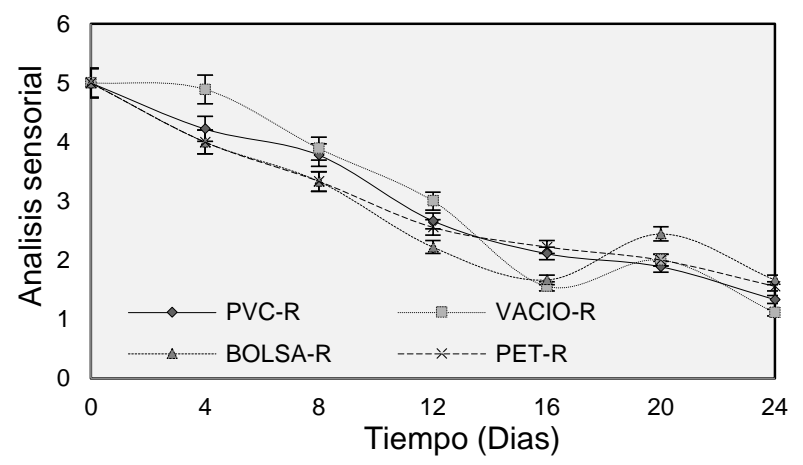

Fig. 1: Notas sensoriales de piña MP, con recubrimiento comestible y 4 tipos de envase, almacenada a $5 \pm 1^{\circ} \mathrm{C}$ y $90 \pm 2 \%$ de $\mathrm{HR}$.

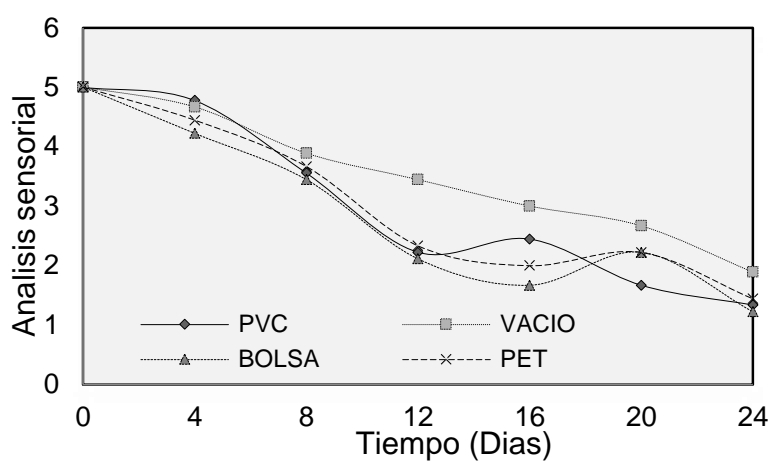

Fig. 2: Notas sensoriales de piña MP, sin recubrimiento comestible y 4 tipos de envase, almacenada a $5 \pm 1^{\circ} \mathrm{C}$ y $90 \pm 2 \%$ de $\mathrm{HR}$.

\section{Análisis de características fisicoquímicas}

En la Figuras 3 y Figura 4, considerando todos los tratamientos, hay una disminución delos valores de pH de la piña MP, iniciando en promedio con un valor de 3,50 y en el día 16 un valor de 2,99, no hubodiferencia estadística $(p<0,05)$ entre los frutos $C R$ y SR.Basados en la estabilidad de los alimentos, los valores finales de $\mathrm{pH}$ son adecuados por ser inferiores a 4,0 (Azeredo, 2004). La aplicación de los ácidos orgánicos en piña MP es el factor causante de la reducción de pHsegún Antoniolli et al., (2012).

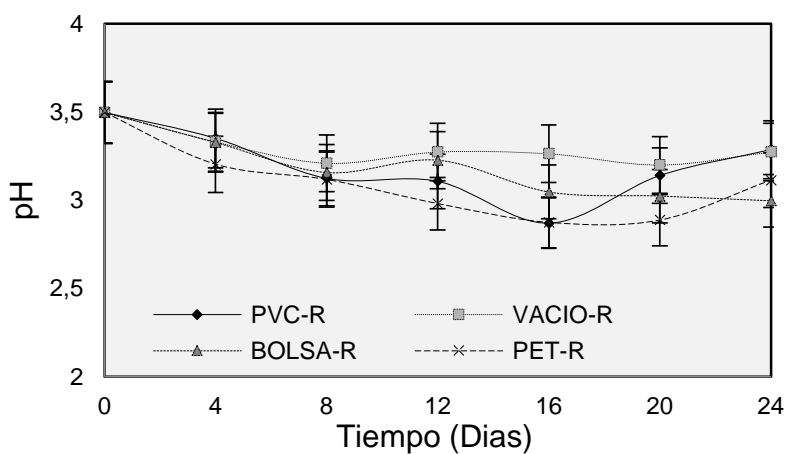

Fig. 3: Valores de $\mathrm{pH}$ depiña MP, con recubrimiento comestible y 4 tipos de envase, almacenada a $5 \pm 1^{\circ} \mathrm{C}$ y $90 \pm 2 \%$ de $\mathrm{HR}$.

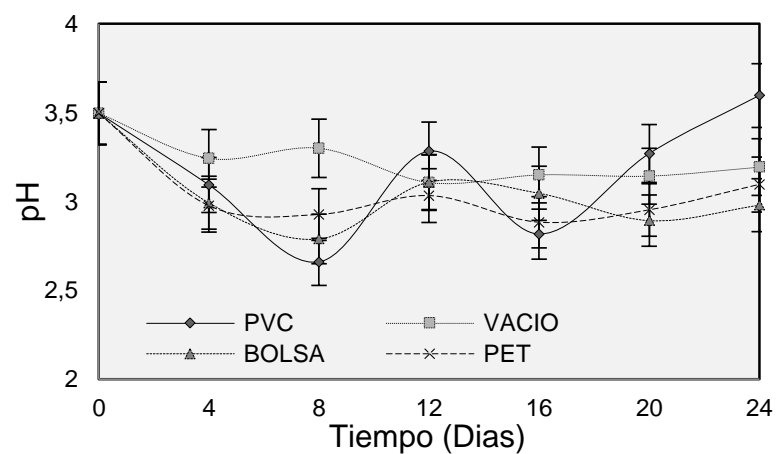

Fig. 4: Valores de $\mathrm{pH}$ de piña MP, sin recubrimiento comestible y 4 tipos de envase, almacenada a $5 \pm 1^{\circ} \mathrm{C}$ y $90 \pm 2 \%$ de $\mathrm{HR}$.

La oscilación del pH en el tiempo tambiénse debe a que las muestras tratadas provenían de diferentes partes de la piña existiendo una diferencia en la maduración de la zona basal y la apical de la piña(Torriet al., 2010). La región basal de la piña siempre muestra valores más altos que la zona media y apical del fruto (Antoniolliet al., 2011). El calcio también interviene en la reducción del pH (Antoniolli et al., 2011), así como la acumulación de $\mathrm{CO}_{2}$ en el ambiente interno del empaque(Rico et al., 2007).Silva et al. (2005), afirma que hay una mayor variación del pH y AT cuando hay presencia de microorganismos en piña 'Pérola' MP.

Las Figuras 5 y Figura 6 muestran el ascenso de la AT de 0,18\% en el primer dia a 0,65\% en el 4 diade almacenamiento, fenómeno explicado por el efecto de los factores discutidos que provocan disminución en los valores de $\mathrm{pH}$. En el día 16 de almacenamiento todos los tratamientos presentaron un valor promedio de AT de $0,64 \%(p<0,05)$. En la Figura 7 y Figura 8 se observa disminución considerable de los valores de sílidos solubles del fruto durante el almacenamiento, iniciando con un valor promedio de 10,67ㅁix y al día 16 un valor de $8,46^{\circ}$ Brix y $8,16^{\circ}$ Brix con y $\sin$ recubrimiento respectivamente. Similar comportamiento encontraron Bueno et al. (2005), un descenso significativo en los sólidos solubles a partir del día 2 y un incremento significativo de la AT en piña MP después del día 8 , a $5^{\circ} \mathrm{C}$ en refrigeración. El tratamiento que exhibió el mayor valor de sólidos solubles al día 16 fue el VACIO sin recubrimiento con un valor de $9,13^{\circ}$ Brix. Valores elevados de sólidos solubles se traduce en sabor más dulce en los frutos (Kader, 2002).La reducción de los sólidos solubleses probablemente debida a la continuación del proceso respiratorio que implica un mayor consumo de sustrato orgánico, es decir, azúcares (Kader, 2002).

Los valores del IM de piña MP, mostraron una redución en el almacenamiento refrigerado. Esta reducciónse debe principalmente a la disminución de la glucosa y fructosa, que indican la senescencia de la fruta 
durante el almacenamiento y encontraron valores del IM entre el 10\% y el 20\%(Rocculi et al.,2009).En este estudio, los mayores valores de IM en piña MP fueron presentados en el tratamiento al VACIO con y sin recubrimiento con un valor respectivo de 20,28 y $19,71(p<0,05)$.

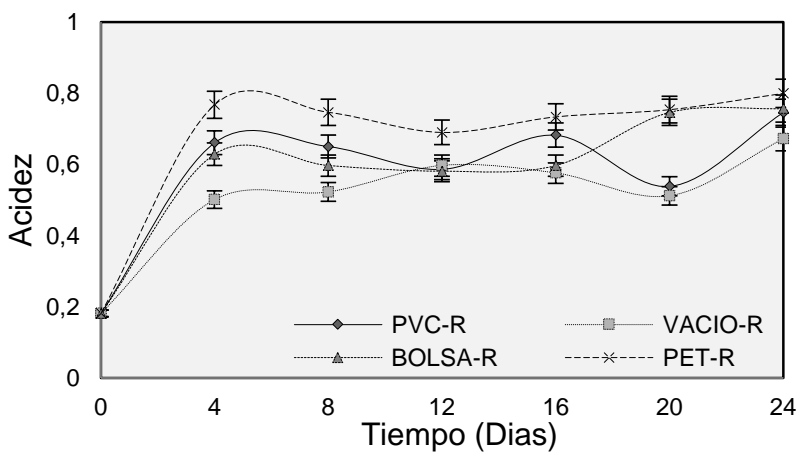

Fig. 5: Valores de acidez titulablede piña MP, con recubrimiento comestible y 4 tipos de envase, almacenada a $5 \pm 1^{\circ} \mathrm{C}$ y $90 \pm 2 \%$ de $\mathrm{HR}$.

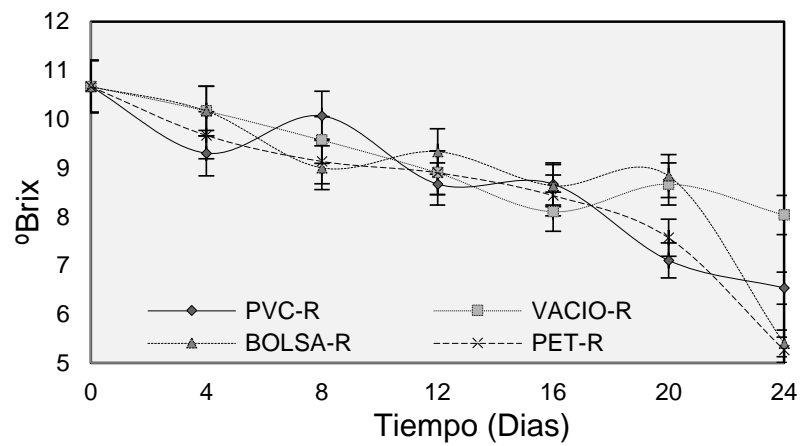

Fig. 7: Valores de sólidos solubles de piña MP, con recubrimiento comestible y 4 tipos de envase, almacenada a $5 \pm 1^{\circ} \mathrm{C}$ y $90 \pm 2 \%$ de $\mathrm{HR}$.

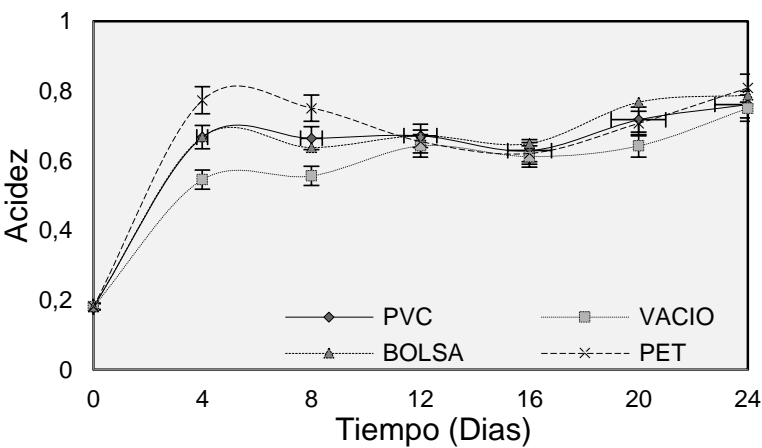

Fig. 6: Valores de acidez titulablede piña MP, sin recubrimiento comestible y 4 tipos de envase, almacenada a $5 \pm 1^{\circ} \mathrm{C}$ y $90 \pm 2 \%$ de HR.

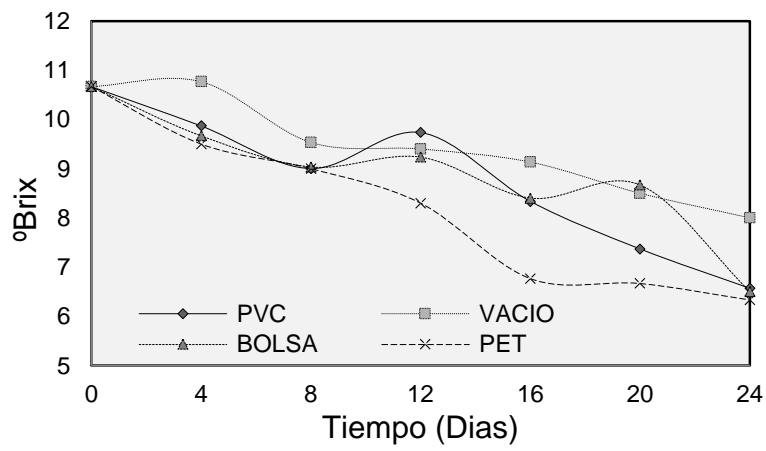

Fig. 8: Valores de sólidos solubles de piña MP, sin recubrimiento comestible y 4 tipos de envase, almacenada a $5 \pm 1^{\circ} \mathrm{C}$ y $90 \pm 2 \%$ de HR.

\section{CONCLUSIONES}

La condición agroindustrial que permite conservar los atributos físico-químicos y sensoriales de piña 'Manzana' mínimamente procesada hasta por 16 díasde almacenamiento refrigerado,consiste en el acondicionamiento al vacío en bolsa plástica de PEBD $70 \mu \mathrm{m}$ a $5 \pm 1^{\circ} \mathrm{C}$ y $90 \pm 2 \%$ de $\mathrm{HR}$, sin recubrimiento comestible y tratada previamente con ácido ascórbico (1\% v/v), ácido cítrico $(1 \% \mathrm{v} / \mathrm{v})$ y $\mathrm{CaCl}_{2}(1 \% \mathrm{v} / \mathrm{v})$. La aplicación del recubrimiento comestible a base de almidón de yuca, cera de carnauba, glicerol y aceite de canola no fue la mejor condición agroindustrial en la conservación de piña mínimamente procesada. Cuando la piñamínimamente procesadaes tratada con recubrimiento comestible y acondicionada al vacío permite conservar los atributos de calidad de hasta por 12 díasa $5 \pm 1^{\circ} \mathrm{C}$ y $90 \pm 2 \%$ de $\mathrm{HR}$.

\section{REFERENCIAS}

Antoniolli, L., Benedito, B., Moreira de Sousa Filho eMen de S. Efeito do cloreto de cálcio na qualidade de abacaxi“ Pérola” minimamente procesado. Pesquisa Agropecuária Brasileira, 38(9), 1105-1110. (2011).

Antoniolli, L. R., Benedetti, B. C., Sá, M.\& Souza, M. Shelf life of minimally processed pineapples treated with ascorbic and citric acids. Bragantia. Campinas, 71(3,), 447-453. (2012).

Azeredo, H. M. C. 2004. Fundamentos de estabilidade de alimentos. 1a. Ed. Fortaleza, Brasil: Embrapa, 195p.

Bueno, S., J. C., Vilas Boas, E. V. D. B., Mônica Elisabeth, T. P., \& Marques Pinheiro, A. C. Avaliação da qualidade do abacaxi "pérola" minimamente processado armazenado sob atmosfera modificada. Ciência $e$ Agrotecnologia, 29(2), 353-361 (2005).

Djioua, T. y otros cinco autores, Improving the storage of minimally processed mangoes (Mangifera indica L.) by hot water treatments. Postharvest Biology and Technology, 52(2), 221-226 (2009). 
Dussán-Sarria, S., Torres-León, C., Hleap-Zapata, J. I. 2014. Efecto de un recubrimiento comestible y diferentes empaques durante el almacenamiento refrigerado de Mango 'Tommy Atkins' mínimamente procesado, Inf. Tecnol. 25(4), 123-130 (2014).

Ediriweera, S., Abeywickrama, K., y Latifah, M.. Effect of Chemical Pretreatments on the Quality of Minimally Processed Pineapple Stored in Polystyrene Packages. Ceylon Journal of Science, 41(2), 151-155. (2012).

Eduardo, M.P., Benedetti, B., y Ferraz, A. Avaliação de índices de firmeza para abacaxi minimamente processado em fatias tratadas com soluções de sais de cálcio. Eng. Agrí. Jaboticabal, 28(1),154-163 (2008).

FAO. Manejo Poscosecha da la Piña. Retrieved from http://www.fao.org/inpho_archive/content/ documents/vlibrary/ac304s/ac304s03.htm. acceso19 de marzo 2013 (2006).

FAO. Producción - Cultivos. FAOSTAT (2014), http://faostat.fao.org/. Acesso: 25 de Abril (2014).

González-Aguilar, G., Ruiz, S., Cruz, R., Rodríguez, A, \& Wang, C. . Physiological and quality changes of fresh-cut pineapple treated with antibrowning agents. LWT - Food Sc. and Technol., 37(3), 369-376. (2004).

ICONTEC NTC 4102. Frutas frescas. Piña manzana. Especificaciones. Colombia (1997).

ICONTEC NTC 4592. Productos frutas y verduras determinación de pH. Bogota (1999a).

ICONTEC NTC 4623. Productos frutas y verduras determinación de la acidez titulable. Colombia (1999b).

ICONTEC NTC 4624. Jugo de frutas y hortalizas. Determinación del contenido de sólidos solubles. Método refractimétrico. Colombia (1999c).

Kader, A.A., 2002. Post-harvest technology of horticultural crops. Oakland: University of California, Division of Agriculture and Natural ResourcesPublication. 3311, 535 p.

Marrero, A., y Kader, A., Optimal temperature and modified atmosphere for keeping quality of fresh-cut pineapples. Postharvest Biology and Technology,39(2), 163-168 (2006).

Martín, O., y Oms, G. (2005). Efecto de la atmósfera modificada en las características físico-químicas y nutricionales de la fruta fresca cortada. Simposium "Nuevas tecnologías de conservación y envasado de frutas y hortalizas. Vegetales frescos cortados" La Habana, Cuba, 47-58.

Rico, D., Martín-Diana, A. B., Barat, J. M., y Barry-Ryan, C. Extending and measuring the quality of fresh-cut fruit and vegetables. Food Science \& Technology, (en linea) 18(7), 373-386 (2007).

Rocculi, P. y otros cuatro autores, Effect of 1-MCP treatment and N2O MAP on physiological and quality changes of fresh-cut pineapple. Postharvest Biology and Technology, 51(3), 371-377. (2009).

Rahman, M. S. Manual de conservacion de alimentos. 2 edicion. ACRIBIA, S.A. pp 190-193 ZaragozaEspaña. (2003).

Rinaldi, M. M.; Benedetti, B.C. ; Sarantopoulos, C.I. G.De L.; Moretti, C.L. .Estabilidade de repolho minimamente processados ob diferentes sistemas de embalagens. Ciência e Tecnologia de Alimentos, Campinas, 29(2), 310-315. (2013).

Saavedra, J. y otros seis autores, Effect of Antioxidants in Fresh Cut Radishes During the Cold Storage. Brazilian Archives of Biology and Technology, 51(December), 1217-1223. (2008).

Saavedra, N. H., y Algecira, N. A. Evaluación de películas comestibles de almidón de yuca y proteína aislada de soya en la conservación de fresas. NOVA, (en linea) 8(14), 171-182 (2010).

Sanjinez-Argandoña, E. J., Branco, I. G., Takito, S. Y., \& Corbari, J. Influencia de la deshidratación osmótica y de la adición de cloruro de calcio en la conservación de kiwis minimamente procesados. Food Science and Technology (Campinas),30, 205-209. (2010).

Silva, G. C. y otros seis autores, Efeito do tipo de corte nas características físico-químicas do abacaxi pérola minimamente processado. Rev. Brasileña de Tecnología de los Alimentos, 25(2), 223-228 (2005).

Torri, L., Sinelli, N., Limbo, S.. Shelf life evaluation of fresh-cut pineapple by using an electronic nose. Postharvest Biology and Technology, 56(3), 239-245. (2010). 